\title{
Choreoathetosis, hypothyroidism, and pulmonary alterations due to human NKX2-1 haploinsufficiency
}

\author{
Heiko Krude, ${ }^{1}$ Barbara Schütz, ${ }^{1}$ Heike Biebermann, ${ }^{1}$ Arpad von Moers, ${ }^{2}$ Dirk Schnabel, ${ }^{1}$ \\ Heidi Neitzel, ${ }^{3}$ Holger Tönnies, ${ }^{3}$ Dagmar Weise, ${ }^{4}$ Antony Lafferty, ${ }^{5}$ Siegfried Schwarz, ${ }^{6}$ \\ Mario DeFelice, ${ }^{7}$ Andreas von Deimling, ${ }^{8}$ Frank van Landeghem, ${ }^{8}$ Roberto DiLauro, ${ }^{7}$ \\ and Annette Grüters ${ }^{1}$
}

${ }^{1}$ Department of Pediatric Endocrinology,

${ }^{2}$ Department of Pediatric Neurology, and

${ }^{3}$ Department of Medical Genetics, Otto Heubner Center for Pediatrics, Berlin, Germany

${ }^{4}$ Altonaer Kinderkrankenhaus, Hamburg, Germany

${ }^{5}$ Department of Pediatrics, Monash University, Clayton, Australia

${ }^{6}$ Department of Experimental Pathology, University of Innsbruck, Innsbruck, Austria

${ }^{7}$ Stazione Zoologica, Napoli, Italy

${ }^{8}$ Department of Neuropathology, Charite-University Hospital, Humboldt University, Berlin, Germany

Address correspondence to: Annette Grüters, Otto-Heubner-Centrum for Pediatrics, Charite,

Humboldt-University, Augustenburgerplatz 1, D-13353 Berlin, Germany.

Phone: 49-30-450-566251; Fax: 0049-30-450-566936; E-mail: annette.grueters@charite.de.

Heiko Krude and Barbara Schultz contributed equally to this work.

Received for publication on October 10, 2001, and accepted in revised form on January 8, 2002.

The occurrence of neurological symptoms and developmental delay in patients affected by congenital hypothyroidism $(\mathrm{CH})$ has been attributed to the lack of thyroid hormone in the developing CNS. Accordingly, after the introduction of neonatal screening programs for $\mathrm{CH}$, which allowed early and adequate treatment, an almost normal outcome for most $\mathrm{CH}$ patients could be achieved. However, a few patients did not reach this favorable outcome despite early and adequate treatment. Here we describe five patients with variable degrees of $\mathrm{CH}$ who suffered from choreoathetosis, muscular hypotonia, and pulmonary problems, an association of symptoms that had not been described before this study. Since this clinical picture matched the phenotype of mice targeted for deletion of the transcription factor gene $N k x 2-1$, we investigated the human $N K X 2-1$ gene in these five patients. We found heterozygous loss of function mutations in each of these five patients, e.g., one complete gene deletion, one missense mutation (G2626T), and three nonsense mutations (2595insGG, C2519A, C1302A). Therefore, the unfavorable outcome in patients with $\mathrm{CH}$, especially those with choreoathetosis and pulmonary symptoms, can be explained by mutations in the NKX2-1 gene rather than by hypothyroidism. Moreover, the association of symptoms in the patients with NKX2-1 mutations points to an important role of human NKX2-1 in the development and function of thyroid, basal ganglia, and lung, as already described for rodents.

This article was published online in advance of the print edition. The date of publication is available from the JCI website, http://www.jci.org. J. Clin. Invest. 109:475-480 (2002). DOI:10.1172/JCI200214341.

\section{Introduction}

Choreoathetosis is defined as rapid involuntary and slow writhing movements due to congenital or acquired defects of the basal ganglia. Huntington disease represents a late-onset progressive variant of chorea with neurodegeneration of the striatum hereditary chorea was linked to a region on chromosome 14 including the NKX2-1 gene, known to be expressed during basal ganglia development in rodents (2).

The NKX2-1 gene is a member of the $N K$-2 gene family (3), which is related to Drosophila NK2/vnd (ventral nervous system defective) and which is defined by a tyrosine residue at position 54 of the DNA-binding homeodomain (HD). Due to its expression in the thyroid gland, the gene was initially named thyroid transcription factor-1 (TTF-1) (4). As described for other NK2 genes, NKX2-1 follows a complex pattern of expression during mouse embryogenesis that includes transcription in the forebrain, thyroid, and developing lung (5). Developmental defects of these structures were observed in homozygous $N k \times 2-1$-null mice (6) characterized by transformation of pallidum to striatum, a complete absence of the thyroid gland, and hypoplasia of the lung with severe respiratory failure and early death.

Based on the cloning from thyroid cells (4) and the severe phenotype of thyroid dysgenesis in $\mathrm{Nkx} 2-2^{-/-}$mice (6), the human $N K X 2-1$ gene first gained interest as a candidate gene for congenital hypothyroidism $(\mathrm{CH})$. However, so far several studies have 
failed to show NKX2-1 mutations in patients with various types of thyroid dysgenesis (7-9). In contrast, two patients with a large chromosomal deletion spanning the NKX2-1 gene were found to be affected by a variety of symptoms in the CNS, thyroid, and lung $(10,11)$. Therefore, it could be hypothesized that mutations in the human NKX2-1 gene might result in a complex disease including neurological, thyroid, and respiratory problems. We searched for mutations in the NKX2-1 gene in patients with symptoms matching this predicted NKX2-1-deficiency phenotype.

\section{Methods}

Mutational analysis. The entire coding region of the NKX2-1 gene was amplified in one fragment from genomic DNA (forward primer: 5'CGGGAGGCAGTCGATCCCTACTCAG; reverse primer: 5' AGGGCCGGCCCGGCGTCCTCTCACC). Exon 1, 2, and 3 were sequenced with an ABI prism 377 sequencer (Applied Biosystems, Foster City, California, USA) using appropriate primers. Identified mutations were confirmed by sequencing of both alleles after cloning PCR fragments into a TOPO cloning vector (Invitrogen, Groningen, The Netherlands) or by restriction analysis of PCR fragments using $M s c I, B f a I$, and HpyCH4v (NEB, England Biolabs).

Immunobistochemistry. Histological studies were performed using standard techniques for peroxidase staining using a NKX2-1/TTF-1-specific
$\mathrm{Ab}$ generated against rat recombinant antigen (DAKO Corp., Carpinteria, California, USA). The human tissues were from autopsy sections. The rat tissues were prepared without prior extended fixation.

Electromobility shift assay. NKX2-1 HD was cloned from genomic DNA of patient 2 into the PCRT7/CT-TOPO expression, and the correct sequence was confirmed by direct sequencing of the constructs. BL21PlysS cells were chemically transformed and stimulated with isopropyl- $\beta$-D-thiogalactopyranosid (IPTG) to produce an $\mathrm{HD} /$ Flag fusion protein according to standard protocols. Total protein extracts were then purified using a Bio-Rad Econo-Pac S Cartridge (BioRad, München, Germany). An electromobility shift essay (EMSA) was performed using ${ }^{32} \mathrm{P}$-labeled oligo-C (5'-CAC TGC CCA GTC AAG TGT TCT TGA-3') as a probe and mutated oligo-C (5'-CAC TGC CCA GTC ACG CGT TCT TGA-3') as a nonspecific control, as described previously (3). To ensure equivalent amounts of wildtype and mutant HD protein, Western blot analysis was performed using a monoclonal M2 anti-Flag Ab (Sigma, Deisenhofen, Germany) according to the improved manufacturer's recommendations. Equivalent amounts of total protein were ascertained by topping the samples with purified extract from bacteria transformed with the empty vector alone and measurement of the correct protein load with the Bio-Rad protein assay (Bio-Rad).
Three-dimensional model. Wild-type HD protein und/NK2 was modeled with RasMol using the atomic coordinates from the Brookhaven Protein Data Bank (http://www.rcsb.org/index.html).

\section{Results}

In a cohort of 150 children identified in a neonatal screening program for $\mathrm{CH}$, we observed choreoathetosis, neonatal respiratory distress, and frequent severe pulmonary infections in two patients (Table 1).

Cytogenetic studies identified an interstitial deletion of chromosome 14 affecting the chromosomal region (14; $\mathrm{q} 11.2 \mathrm{q} 13.3$ ) in patient 1 (Figure 1), including the NKX2-1 locus confirmed by comparative genomic hybridization (data not shown). In patient 2 , direct sequencing of PCR products spanning the entire coding region of the NKX2-1 gene revealed a heterozygous mutation (G2626T) in exon 3 (Figure 1). This missense mutation changes a highly conserved valine at amino acid (aa) position 45 of the DNA binding HD to phenylalanine (Figure 2a).

Based on these results, we extended the NKX2-1 mutational analysis to other patients with $\mathrm{CH}$ and mild thyroid abnormalities associated with neurological symptoms including choreoathetosis, muscular hypotonia, ataxia, and retarded development, as well as pulmonary symptoms diagnosed in several other centers. Within this group of 13 patients we detected a boy (patient 3, Table 1) with a heterozygous $\mathrm{GG}$ insertion at nucleotide

Table 1

Phenotype of NKX2-1 mutant patients

\begin{tabular}{|c|c|c|c|c|c|c|c|c|}
\hline Patient & $\begin{array}{c}\text { Current age } \\
\text { [years] }\end{array}$ & $\begin{array}{c}\text { Max. TSH } \\
{[\mathrm{mU} / \mathrm{I}]} \\
(0.5-5)\end{array}$ & $\begin{array}{c}\text { Min. T4 } \\
{[\mu \mathrm{g} / \mathrm{dl}]} \\
(6.5-15)\end{array}$ & $\begin{array}{l}\text { Thyroid gland } \\
\text { imaging }\end{array}$ & $\begin{array}{c}\text { Respiratory } \\
\text { distress }\end{array}$ & $\begin{array}{l}\text { Pulmonary } \\
\text { infection }\end{array}$ & Choreoathetosis & Mutation \\
\hline 1 & 16 & 44 & 5.9 & hypoplasia & severe & severe-frequent & severe & deletion \\
\hline 2 & 15 & 122 & 2.9 & Bhypoplasia & moderate & severe-frequent & severe & $\begin{array}{c}\text { G2626T } \\
\text { (missense) }\end{array}$ \\
\hline 3 & 3 & 12 & 11 & normal & no & few-mild & severe & $\begin{array}{l}\text { 2595insGG } \\
\text { (nonsense) }\end{array}$ \\
\hline 4 & 13 & 55 & 3.9 & Bhypoplasia & no & no & severe & $\begin{array}{c}\text { C2519A } \\
\text { (nonsense) }\end{array}$ \\
\hline 5 & 1.5 & 825 & $<1$ & Cagenesis & severe & no & $\begin{array}{l}\text { Dmuscle } \\
\text { hypotonia }\end{array}$ & $\begin{array}{c}\text { C1302A } \\
\text { (nonsense) }\end{array}$ \\
\hline
\end{tabular}

ATSH concentrations of patients 1, 2, 4, and 5 are from the initial diagnosis in the neonatal period or from the off-treatment period at an age of 2 years. Patient 2 was diagnosed with euthyroid hyperthyreotropinemia at an age of 15 months. All TSH concentrations during the period of L-thyroxine treatment were normal, indicating adequate substitution. ${ }^{\mathrm{B} N o}$ apparent thyroid tissue seen in scintigraphy performed in neonatal period; hypoplasia at reinvestigation by ultrasound. CSo far only scintigraphy performed in neonatal period. ${ }^{\mathrm{D}}$ Muscular hypotonia represents the initial symptom of infants developing choreoathetosis later in childhood. 


\section{Figure 1}

Genotype of NKX2-1-deficient patients. The complete genomic structure of NKX2-1 gene is shown. Different transcription start sites have been described leading to $5^{\prime}$-alternative transcripts indicated by +1 in black, according to Hamdan et al. (25), and in gray, according to lkeda et al. (26). Numbering of nucleotides are shown according to both references and have been used to indicate the mutations according to the most extended genomic sequence reported by Hamdan et al. (25). Numbering of a residues are relative to the first residue of the DNA-binding $\mathrm{HD}$ to achieve the best comparison between mutations in different HDs containing transcription factors, as suggested by the terminology of vnd/NK2 gene mutations (13). Mutations are indicated as arrows. If possible mutations were confirmed by restriction enzyme analysis, as shown for patients 3, 4, and 5, parental samples were obtained for patient 2 (sequence data show normal results, not shown) and patient 3 ; ctr, control DNA; nc, non-cut DNA; M, mother; $\mathrm{F}$, father; $\mathrm{P}$, patient.

position 2595 in exon 3 (Figure 1). He was affected predominantly by choreoathetosis and had only mild thyroid dysfunction with elevated thyroid-stimulating hormone (TSH) and normal serum thyroid hormone concentrations. The mutation results in a frameshift that leads to a truncated protein lacking the entire third helix of the HD (Figure 2). In a fourth male patient with $\mathrm{CH}$ and choreoathetosis, a heterozygous point mutation (C2519A) in exon 3 changed Ser at position 9 of the HD to a premature stop mutation. The fifth patient was identified with severe $\mathrm{CH}$ associated with muscular hypotonia and was affected by severe respiratory distress after birth, requiring oxygen administration for several weeks. A nonsense mutation in exon 2 was found (C1320A) changing Cys at position -75 relative to the HD into a premature stop codon (Figure 1). The clinical data of all five patients are summarized in Table 1. All described mutations were heterozygous, and available parental DNA of patient 2 and 3 were normal, suggesting de novo mutations, at least in these two patients (Figure 1).

For a better understanding of the functional consequences of the muta-

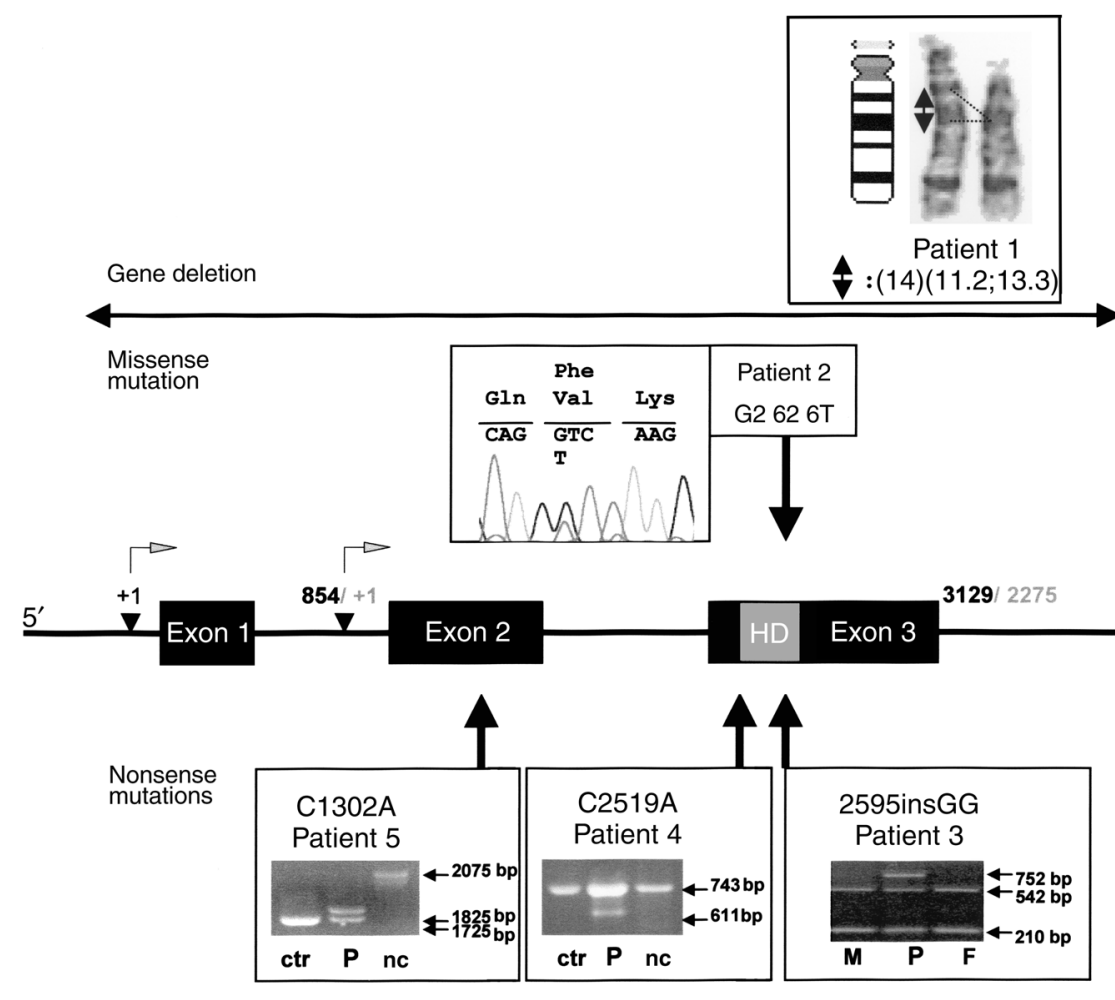

tions we generated a three-dimensional computer model using the published coordinates of the NK2/vndDNA complex (Figure 2b) (12). According to this model, the change of the highly conserved valine 45 to phenylalanine predicts a marked distortion of the HD/DNA complex as already described for the adjacent alanine 35 threonine mutation found in a lethal Drosophila NK2/vnd allele (13). EMSAs confirmed this predicted loss of binding of the mutant HD (Figure 2 , c and d). Furthermore, according to this model, the probable loss of the entire binding helix 3 due to the nonsense mutation of patient 3 (2595insGG) seems to result in a complete loss of function of the NKX2-1 gene product (Figure 2e). The same effect can be expected for the loss of the entire HD due to the nonsense mutation in patients 4 and 5 .

To define more precisely the phenotype of patients with NKX2-1 mutations we reevaluated the structural and functional defects of the NKX2-1-deficient patients in more detail. Cranial magnetic resonance imaging (MRI) with inversion recovery imaging in patient 2 revealed changes of the basal ganglia, including a pallidum with reduced size compared with normal values and without visible division into a lateral and medial part (Figure 3, a and b). In addition, in patient 1 and 2 an identical cystic mass was found in a position craniodorsal to the pituitary (Figure 3, c and d). At follow-up no progression of the cystic mass was evident. Functional tests revealed a normal secretion of pituitary hormones (data not shown).

The clinical data suggested a defect of basal ganglia due to NKX2-1 deficiency. Since it has already been described that NKX2-1 is expressed during embryogenesis in the pallidum, a developmental defect can be assumed. In addition, we asked if NKX2-1 is also expressed in adult tissue, which would imply an additional functional defect of the basal ganglia. Therefore, expression of the NKX2-1 protein in adult tissues derived from human and rats was investigated. By immunohistochemistry, nuclear staining was only visible in the human lung (Figure 4b), human thyroid (Figure 4c), rat lung (Figure 4e), and rat hypothalamus (Figure 4f). In contrast, NKX2-1 staining was negative in adult human (Figure 4a) as well as rat pallidum (Figure 4d). 


\section{Figure 2}

Position of aa exchange due to the NKX2-1 mutations and structural consequences for the DNA interaction. (a) Alignment of the nine known mammalian NK2 HDs $(3,27)$; Tyr54, which is characteristic for NK2 HDs, is shown in white, mutations detected in our patients are shown in white italic underlined, the known missense mutations of the NKX2-5 HD are shown in gray (20). (b) Three-dimensional model of NK2 HD-DNA complex. Position of Val45Phe mutation in patient 2 is shown in black. (c) EMSA performed with in vitro-expressed HD protein show a clear loss of binding capacity in the mutant. Lane 1: without protein, lanes 2-5: wild-type protein $(0.5,1,2$, and $5 \mu$ l purified protein) lane 6: unrelated oligo; lanes 7 and 8: competition with 100-fold excess of unlabeled oligo-C (lane 7 ) and unrelated oligo (lane 8) (amount of protein applied corresponding to lane 3); lanes 9-11: mutant protein (2, 5, and $10 \mu \mathrm{l}$ of purified protein); lane 12: mutant protein with unrelated and unlabeled oligo; black arrow marks bound oligo, white arrow marks free oligo. (d) Western blots using anti-Flag-antibody are aligned with lanes 2-5 and 9-11 of (c) showing the equivalence in amounts of HD protein used.(e) The truncated HD due to the 2595insGG nonsense mutation in patient 3 is shown in the predicted conformation relative to the DNA-binding groove lacking helix 3. a

\begin{tabular}{|c|c|}
\hline fruitfly & Nk2/vnd \\
\hline mutant & $\mathrm{Nk} 2$ / vnd \\
\hline human & NKX2-1 \\
\hline patient2 & $N K \times 2-1$ \\
\hline patient3 & NKX $\times 2-1$ \\
\hline mouse & Nkx2-1 \\
\hline mouse & Nkx2-2 \\
\hline mouse & Nkx2-3 \\
\hline human & $N k \times 2-4$ \\
\hline mouse & Nkx $2-4$ \\
\hline human & NKX2-5 \\
\hline mutants & NKX2-5 \\
\hline mouse & Nkx2-5 \\
\hline mouse & Nkx2-6 \\
\hline fish & nkx2-7 \\
\hline chicken & Nkx2-8 \\
\hline mouse & $N k \times 2-9$ \\
\hline
\end{tabular}
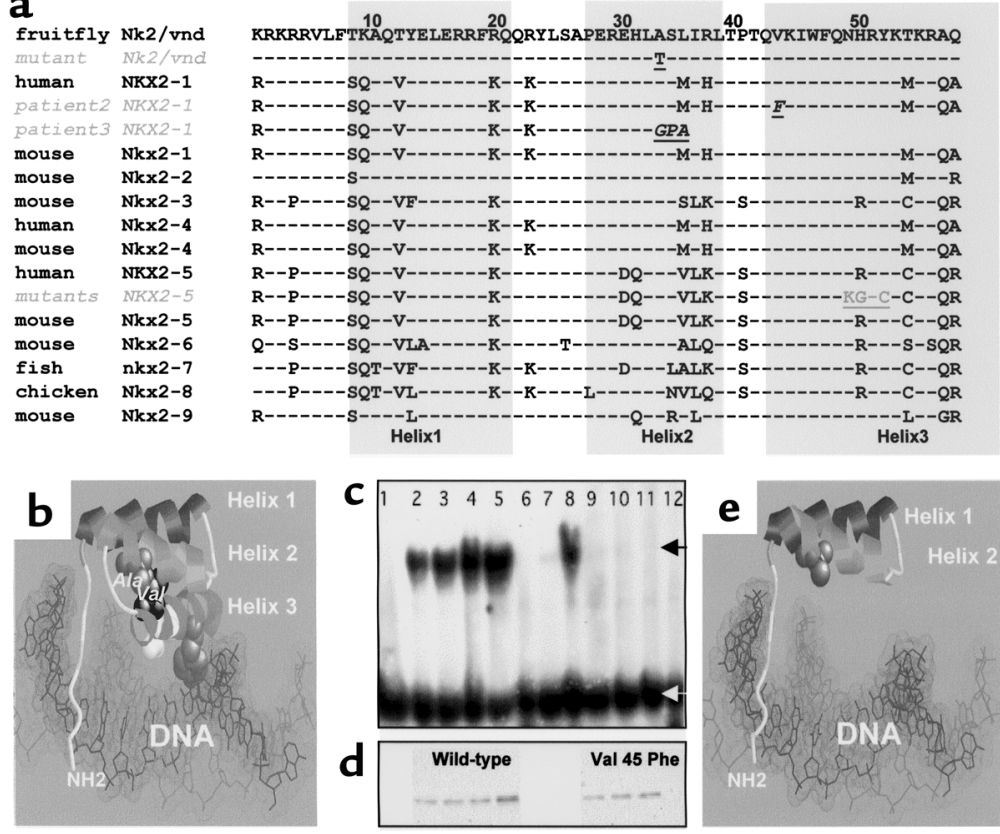

\section{Discussion}

We identified the first five patients with heterozygous NKX2-1 mutations who are affected by choreoathetosis, hypothyroidism, and respiratory problems, an association of symptoms as yet not described. Since all five patients were adequately treated with L-thyroxine, the unfavorable neurological outcome is most likely related to NKX2-1 deficiency in the thyroid and the brain rather than to the consequence of hypothyroidism. Therefore, the observation of a developmental delay or neurological symptoms in $\mathrm{CH}$ patients diagnosed in neonatal screening programs should lead to a molecular diagnosis of genes expressed in the developing thyroid and brain.

The finding of dysgenesis of the pallidum in patient 2 corresponds to developmental defects of the basal ganglia in Nkx2-1-deleted mice (14). In addition, the structural alteration of the basal ganglia in patient 2 seems to explain the clinical finding of choreoathetosis. Since immunohistochemical studies revealed that the NKX2-1 gene product is not expressed in adult neurons of the basal ganglia, NKX2-1 seems to be of importance in the developmental regulation of basal ganglia formation, but does not play a role in continuous basal ganglia function.

This predominant role for CNS organogenesis rather than CNS function was also suggested by the finding of a cystic diencephalic mass in patient 1 and 2. Nkx2-1 is expressed in the diencephalic endoderm leading to subsequent expression of Fgf8, which in turn promotes pituitary development (15). In addition, homozygous targeted disruption of $\mathrm{Nkx} 2-1$ results in ablation of the pituitary gland (6). This functional role of $\mathrm{Nkx} 2-1$ at the interphase of diencephalic and pituitary development in rodents correlates with the formation of a cystic mass exactly at the same position in affected patients. Since in the heterozygous patients the morphology of the pituitary appeared to be normal and pituitary hormone secretion was not altered, these findings suggest that the cystic mass reflects an alteration of diencephalic development rather than pituitary function.

The variable spectrum of thyroid abnormalities in the NKX2-1-mutant patients includes functional as well as developmental defects. While in patient 3 the mild elevation of TSH with normal thyroid hormone secretion clearly points to a functional defect only, the finding of thyroid dysgenesis in the other four patients also reflects a developmental defect of the thyroid gland. A functional thyroid defect can be explained by the reduced activation of the well-known NKX2-1 target genes in thyroid follicular cells, e.g., TSH receptor, thyroid peroxidase, and thyroglobulin gene (16). The molecular defects leading to thyroid dysgenesis in the NKX2-1 mutation carriers remains elusive since no target genes of NKX2-1 during thyroid development has been described.

Nkx2-1 was shown to participate both in lung morphogenesis and in respiratory epithelial cell gene regulation, especially of surfactant protein (SP) genes (17). It has been shown that SP-B is critical for reduction of alveolar resistance leading to respiratory distress in SP-B $\mathrm{B}^{-/-}$mice (18). SP-A and -D play an important role in pulmonary host defense (19). Variable pulmonary problems were found in all patients, including neonatal respiratory distress requiring mechanical ventilation (for a short time in patients 1 and 2 and for several weeks in patient 5). In addition, frequent and sometimes severe pulmonary infections in the first years of life were present (patient 1 and 2 ). In patient 1 , 


\section{Figure 3}

Morphological brain alterations of patients with NKX2-1 mutations. (a) Inversion recovery view of MRI sequence of supratentorial structures in a normal age-matched proband. White matter maturation is complete; basal ganglia appear hypointense in comparison. Medial and lateral parts of the pallidum are clearly distinguishable. c, caudate; $p$, putamen; Ip, lateral part; mp, medial part. (b) MRI of patient 2 demonstrates abnormal basal ganglia. At a comparable level of MRI section a hypoplastic pallidum with a lack of differentiation into medial and lateral part can be seen (arrow). Structure and signal intensity of putamen and caudate nucleus are normal. Brain maturation is unaltered, and there is no malformation of cerebral cortical structure. (c) MRI of the pituitary-diencephalic region of patient 1 (T1-image) showing a cystic mass in the posterior part of the sella turcica (arrow). (d) MRI of this region of patient 2 (T2-image) showing an identical mass at the same position (arrow). Isodense appearance in respect to ventricular structures clearly demonstrates its cystic character.

a developmental defect appearing as lung sequestration was diagnosed and resected within the first year of life. Therefore, alteration of NKX2-1 function during development could have resulted in the lung sequestration of patient 1 , whereas the predisposition to pulmonary infections and neonatal respiratory distress can be explained on a molecular level by the reduced expression of SPs.

The detailed description of defects in homozygous Nkx2-1-null mice show a more severe phenotype compared with the symptoms observed in the heterozygous human patients. Moreover heterozygous mice were not reported to have any abnormalities (6). The same differences in human versus mice phenotypes were found in another member of the NK2 gene family, e.g., $N K X 2-5$. In humans carrying a heterozygous $N K X 2-5$ mutation an atrioventricular conduction block associated with mild to severe morphological
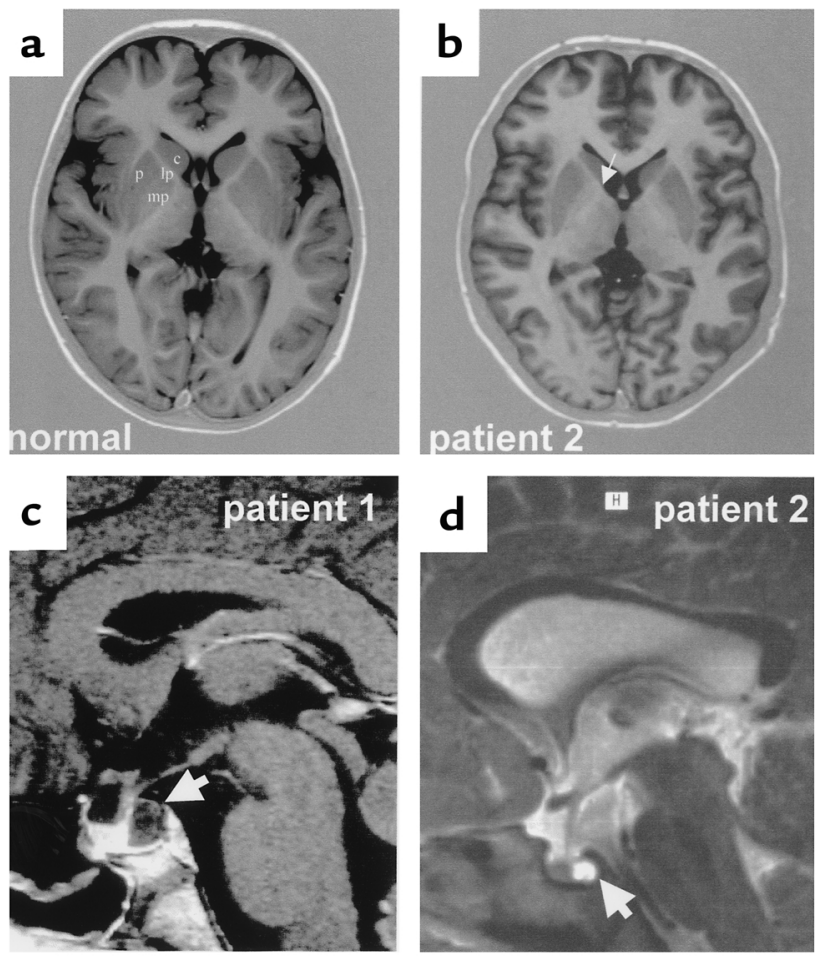

cardiac defects was described (20). In contrast, heterozygous $\mathrm{Nkx} 2-5^{+/-}$mice were reported to be normal while in NKX2-5/- mice an arrest of heart development at an early stage was reported (21). Subsequent reinvestigation of heterozygous Nkx2-5 mice revealed subtle functional and morphological cardiac defects influenced by the genetic background (22). Similar findings in heterozygous NKX2-1 mice have now been reported (23). This dominant inheritance of transcription factor mutations with species-specific sensitivity to gene dosage has already been described for members of other transcription factor gene families involved in early development, such as PAX genes (24)

Our data show that NKX2-1 mutations result in a complex phenotype resembling the tissue expression pattern of the NKX2-1 gene, affecting brain, thyroid, and lung. This pattern of organ manifestation of NKX2-1 mutations appears to be similar in the affected patients. This clinical similarity in patients affected by a large deletion of chromosome $14 \mathrm{q}$ and by loss of function mutations within the NKX2-1 gene implies that this phenotype is entirely due to NKX2-1 haploinsufficiency. The

\section{Figure 4}

Expression of the NKX2-1 protein in human and rat adult tissues. (a) Human basal ganglia. The inset shows no nuclear staining of the NKX2-1 Ab. Nuclear staining is visible in human lung (b) and human thyroid (c). In the rat brain (d) staining is negative in the pallidum (as shown in the inset) on a cellular level but positive in the lung $(\mathbf{e})$ and the hypothalamus (f). pu, putamen; pa-I, lateral pallidum; pa-m, medial pallidum; ic, internal capsle.
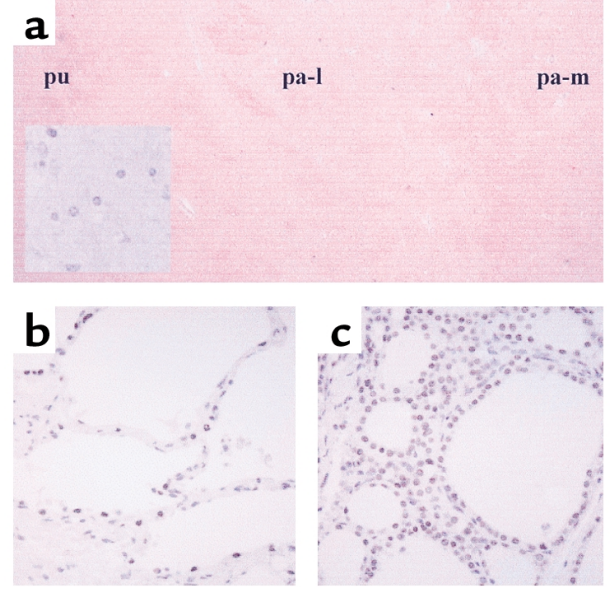
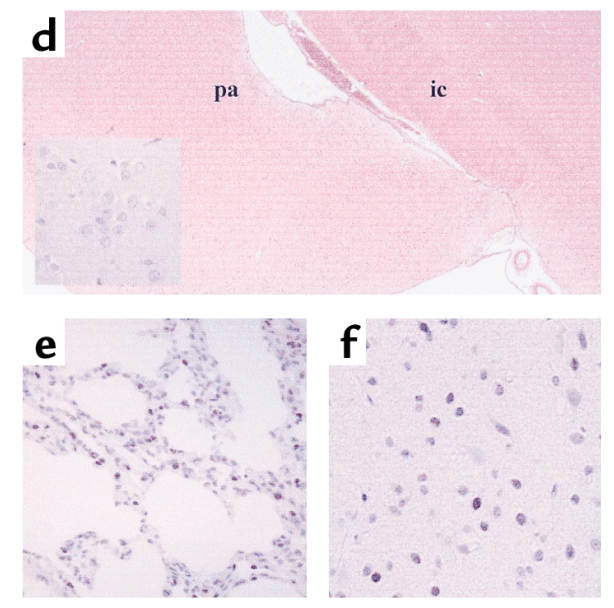
spectrum of developmental and functional defects further point to a dual role of NKX2-1 in organogenesis as well as in gene expression in adult tissues. The finding of dysplasia of the pallidum associated with choreoathetosis in NKX2-1-deficient patients represents, to our knowledge, the first monogenetic defect leading to a developmental disorder of basal ganglia. The prominent finding of nonprogressive choreoathetosis in NKX2-1-deficient patients may suggest that other congenital extrapyramidal movement disorders could be caused by NKX2-1 mutations. This is supported by recent link of benign hereditary chorea to a region on chromosome 14 including the NKX2-1 gene locus (2).

\section{Acknowledgments}

We thank K. Huhne, P. Ambrugger, and A. Gerlach for technical assistance, and D. Lehmann for providing MRI images of patients 1 and 2. This work was supported by a grant of the Deutsche Forschungsgemeinschaft (SFB 577 A5) and the Sonnenfeld-Stiftung.

1. Hodgson, J.G. 1999. A YAC mouse model for Huntington's disease with full length mutant huntingtin, cytoplasmic toxicity, and selective striatal neurodegeneration. Neuron. 23:181-192.

2. de Vries, B.B., et al. 2000. Benign hereditary chorea of early onset maps to chromosome 14q. Am.J. Hum. Genet. 66:136-142.

3. Harvey, R.P. 1996. NK-2 homeobox genes and heart development. Dev. Biol. 178:203-216.

4. Guazzi, S., et al. 1990. Thyroid nuclear factor 1
(TTF-1) contains a homeodomain and displays a novel DNA binding specificity. EMBO J. 9:3631-3639.

5. Lazzaro, D., Price, M., de Felice, M., and Di Lauro, R. 1991. The transcription factor TTF-1 is expressed at the onset of thyroid and lung morphogenesis and in restricted regions of the foetal brain. Development. 113:1093-1104

6. Kimura, S., et al. 1996. The T/ebp null mouse: thyroid-specific enhancer-binding protein is essential for the organogenesis of the thyroid, lung, ventral forebrain, and pituitary. Genes Dev. 10:60-69.

7. Perna, M.G., et al. 1997. Absence of mutations in the gene encoding thyroid transcription factor-1 (TTF-1) in patients with thyroid dysgenesis. Thyroid. 7:377-381.

8. Hishinuma, A., et al. 1998. Sequence analysis of thyroid transcription factor-1 gene reveals absence of mutations in patients with thyroid dysgenesis but presence of polymorphisms in the $5^{\prime}$ flanking region and intron. Endocr. $J$ 45:563-567.

9. Lapi, P., et al. 1997. Mutations in the gene encoding thyroid transcription factor-1 (TTF-1) are not a frequent cause of congenital hypothyroidism (CH) with thyroid dysgenesis. Thyroid. 7:383-387.

10. Devriendt, K., Vanhole, C., Matthijs, G., and de Zegher, F. 1998. Deletion of thyroid transcription factor-1 gene in an infant with neonatal thyroid dysfunction and respiratory failure. New Engl. J. Med. 338:1317-1318.

11. Iwatani, N., Mabe, H., Devriendt, K., Kodama, M., and Miike, T. 2000. Deletion of NKX2.1 gene encoding thyroid transcription factor- 1 in two siblings with hypothyroidism and respiratory failure. J. Pediatr. 137:272-276.

12. Gruschus, J.M., Tsao, D.H., Wang, L.H., Nirenberg, M., and Ferretti, J.A. 1997. Interactions of the vnd/NK2 homeodomain with DNA by nuclear magnetic resonance spectroscopy: basis of binding specificity. Biochemistry. 36:5372-5380

13. Xiang, B., Weiler, S., Nirenberg, M., and Ferretti, J.A 1998. Structural basis of an embryonically lethal single Ala $\rightarrow$ Thr mutation in the vnd/NK-2 homeodomain. Proc. Nat. Acad. Sci. USA. 95:7412-7416.

14. Sussel, L., Marin, O., Kimura, S., and Rubenstein, J.L. 1999. Loss of Nkx2.1 homeobox gene function results in a ventral to dorsal molecular respecification within the basal telencephalon: evidence for a transformation of the pallidum into the striatum. Development. 126:3359-3370.

15. Takuma, N., et al. 1998. Formation of Rathke's pouch requires dual induction from the diencephalon. Thyroid-specific gene expression. Devel opment. 125:4835-4840.

16. Damante, G., and Di Lauro, R. 1994. Thyroid specific gene expression. Biochim. Biophys. Acta. 1218:255-266.

17. Mendelson, C.R. 2000. Role of transcription factors in fetal lung development and surfactant protein gene expression. Annu. Rev. Physiol. 62:875-915.

18. Clark, J.C., et al. 1995. Targeted disruption of the surfactant protein B gene disrupts surfactant homeostasis causing respiratory failure in newborn mice. Proc. Natl. Acad. Sci. 92:7794-7798.

19. Crouch, E., and Wright, J.R. 2001. Surfactant proteins A and D and pulmonary host defense. Annu. Rev. Physiol. 63:521-554.

20. Schott, J.J., et al. 1998. Congenital heart disease caused by mutations in the transcription factor NKX2-5. Science. 281:108-111.

21. Lyons, I., et al. 1995. Myogenic and morphogenetic defects in the heart tubes of murine embryos lacking the homeobox gene Nkx2-5. Genes Dev. 9:1654-1666.

22. Biben, C., et al. 2000. Cardiac septal and valvular dysmorphogenesis in mice heterozygous for mutations in the homeobox gene $\mathrm{Nkx} 2-5$. Circ. Res. 87:888-895.

23. Pohlenz, J., et al. 2002. Partial deficiency of Thyroid transcription factor 1 produces predominantly neurological defects in humans and mice. J. Clin. Invest. 109:469-473. DOI:10.1172/JCI200214192.

24. Wilm, B., Dahl, E., Peters, H., Balling, R., and Imai, K. 1998. Targeted deletion of Pax 1 defines ist null phenotype and proves haploinsuffiency. Proc. Natl. Acad. Sci. USA. 95:8692-8697.

25. Hamdan, H., et al. 1998. Structure of the human Nkx2.1 gene. Biochim. Biophys. Acta. 1396:336-348.

26. Ikeda, K., et al. 1995. Gene structure and expression of human thyroid transcription factor- 1 in respiratory epithelial cells. J. Biol. Chem. 270:8108-8114.

27. Venkatesh, T., Holland, N., Holland, L., Su, M.T., and Bodmer, R. 1999. Sequence and developmental expression of amphioxus AmphiNk2-1 insights into the evolutionary origin of the vertebrate thyroid gland and forebrain. Dev. Genes Evol. 209:254-259. 